ANNALES

POLONICI MATHEMATICI

$85.3(2005)$

\title{
On the Neumann problem with combined nonlinearities
}

\author{
by JAN Chabrowski (Brisbane) and JiAnfu YANG (Wuhan)
}

\begin{abstract}
We establish the existence of multiple solutions of an asymptotically linear Neumann problem. These solutions are obtained via the mountain-pass principle and a local minimization.
\end{abstract}

1. Introduction. The main purpose of this paper is to investigate the nonlinear Neumann problem

$$
\left\{\begin{array}{l}
-\Delta u=h(x) u^{q}+f(x, u) \quad \text { in } \Omega, \\
\partial u / \partial \nu=0 \quad \text { on } \partial \Omega, \quad u>0 \quad \text { on } \Omega,
\end{array}\right.
$$

where $0<q<1, \Omega \subset \mathbb{R}^{N}, N \geq 3$, is a smooth bounded domain and $\nu$ denotes the unit outward normal to the boundary. We assume that $h$ is a smooth function on $\bar{\Omega}, h(x) \leq 0$ on $\Omega$ with a strict inequality on a set of positive measure, $f(x, s) \geq 0$ for $s \geq 0$ and is asymptotically linear at infinity. Additional assumptions on $f$ and $h$ will be formulated later.

The corresponding Dirichlet problem has been studied by many authors (see [6]-[9], [13], [14], and references given there). Some existence results with $f$ interfering with the eigenvalues of higher order but without a concave term can be found in [2], [11] and [12]. In particular, in [2] a nonlinearity is allowed to be asymptotically linear in $-\infty$. However, it seems that not much attention has been given to problem (1.1).

The main purpose of this paper is to establish the existence of at least two solutions of problem (1.1) (see Theorems 2.2 and 2.3). The first solution is obtained through the mountain-pass principle and second by a local minimization. In these theorems it is assumed that $f$ is asymptotically linear at $-\infty$ and $+\infty$ and that $h$ changes sign. We also consider a nonlinearity which is only asymptotically linear at $+\infty$. In this case we prove the existence of a

2000 Mathematics Subject Classification: 35B33, 35J65, 35Q55.

Key words and phrases: Neumann problem, asymptotically linear nonlinearities, multiple solutions.

Research of J. F. Yang supported in part by NSF of China. 
mountain-pass solution provided $h<0$ on $\Omega$. Theorem 3.2 provides the existence of a solution in the case where $f$ is superlinear via the mountain-pass principle. However, we do not impose the usual Ambrosetti-Rabinowitz condition on $f$. To overcome this difficulty we use some ideas from the papers [8], [13] and [14]. If $h \equiv 0$, then under our assumptions on $f$ problem (1.1) does not have a positive solution. The results of this paper show that a small concave perturbation produces at least two distinct solutions.

We use standard notations. In a given Banach space $X$ weak convergence is denoted by " $\rightarrow$ " and strong convergence by " $\rightarrow$ ". The norms in the Lebesgue spaces $L^{p}(\Omega)$ are denoted by $\|\cdot\|_{p}$. By $|A|$ we denote the Lebesgue measure of a set $A \subset \mathbb{R}^{N}$.

We recall that a $C^{1}$ functional $\Phi: X \rightarrow \mathbb{R}$ on a Banach space $X$ satisfies the Palais-Smale condition at level $c\left((\mathrm{PS})_{c}\right.$ condition for short) if each sequence $\left\{x_{n}\right\} \subset X$ such that $\Phi\left(x_{n}\right) \rightarrow c$ and $\Phi^{\prime}\left(x_{n}\right) \rightarrow 0$, as $n \rightarrow \infty$, in $X^{*}$ is relatively compact in $X$.

2. Mountain-pass structure and local minimization. In this section we assume that

(H1): $\quad h$ changes sign and $\int_{\Omega} h(x) d x<0$,

(F1): $f \in C(\bar{\Omega}, \mathbb{R}), f(x, 0)=0$ for $x \in \Omega, f(x, s)>0$ for $s \neq 0$, and

$$
\lim _{s \rightarrow 0} \frac{f(x, s)}{s}=0, \quad \lim _{s \rightarrow \infty} \frac{f(x, s)}{s}=l_{1}, \quad \lim _{s \rightarrow-\infty} \frac{f(x, s)}{s}=l_{2},
$$

where $l_{1}>0$ and $l_{2}<0$ are constants.

Solutions will be obtained as critical points of the functional

$$
J(u)=\frac{1}{2} \int_{\Omega}|\nabla u|^{2} d x-\frac{1}{q+1} \int_{\Omega} h(x)|u|^{q+1} d x-\int_{\Omega} F(x, u) d x,
$$

where $F(x, t)=\int_{0}^{t} f(x, s) d s$. This functional is $C^{1}$ on $H^{1}(\Omega)$, where $H^{1}(\Omega)$ is the usual Sobolev space for the Neumann problem. The norm of $H^{1}(\Omega)$ is denoted by $\|\cdot\|$ and is given by

$$
\|u\|=\left(\int_{\Omega}\left(|\nabla u|^{2}+u^{2}\right) d x\right)^{1 / 2} .
$$

We shall use the decomposition $H^{1}(\Omega)=\mathbb{R} \oplus V$, where

$$
V=\left\{v ; \int_{\Omega} v d x=0 \text { and } \nabla v \in L^{2}(\Omega)\right\} .
$$

This decomposition follows from the fact that the first eigenvalue of $-\Delta$ with the Neumann boundary conditions is 0 and the corresponding eigenfunctions are constant functions. Using this decomposition we define the following 
norm on $H^{1}(\Omega)$ :

$$
\|u\|_{V}=\left(t^{2}+\int_{\Omega}|\nabla v|^{2} d x\right)^{1 / 2}, \quad u=t+v .
$$

The norms $\|\cdot\|$ and $\|\cdot\|_{V}$ are equivalent. Indeed, if $u \in H^{1}(\Omega)$ and $\int_{\Omega} u(x) d x=0$, then

$$
\|u\|_{2} \leq K(\Omega)\|\nabla u\|_{2}
$$

where $K=K(\Omega)>0$ is a constant depending on $\Omega$. Using (2.1) we establish the following inequality:

$$
\min (1,|\Omega|)^{1 / 2}\|u\|_{V} \leq\|u\| \leq \max \left(1+K^{2},|\Omega|\right)^{1 / 2}\|u\|_{V}
$$

First, we observe that

$$
\|u\|^{2}=\int_{\Omega}\left(|\nabla v|^{2}+v^{2}+t^{2}\right) d x \geq \min (1,|\Omega|)\|u\|_{V}^{2} .
$$

To obtain the right-hand-side inequality of (2.2) we use (2.1):

$$
\begin{aligned}
\|u\|^{2} & =\int_{\Omega}\left(|\nabla v|^{2}+t^{2}+v^{2}\right) d x \leq\left(1+K^{2}\right) \int_{\Omega}|\nabla v|^{2} d x+t^{2}|\Omega| \\
& \leq \max \left(1+K^{2},|\Omega|\right)\|u\|_{V}^{2} .
\end{aligned}
$$

We commence by checking the mountain-pass geometry of $J$. Since $V$ is continuously embedded into $L^{q+1}(\Omega)$ we have the following quantitative statement: there exists a constant $\eta>0$ such that

$$
\|\nabla v\|_{2} \leq \eta|t| \quad \text { implies } \int_{\Omega} h(x)|t+v|^{q+1} d x \leq-|t|^{q+1} \alpha
$$

for every $t \in \mathbb{R}$ and $v \in V$, where $\alpha=-\frac{1}{2} \int_{\Omega} h(x) d x>0$.

Proposition 2.1. There exist constants $\kappa>0, \varrho>0$ and $\gamma>0$ such that

$$
J(u) \geq \kappa \quad \text { for }\|u\|=\varrho
$$

and every function $h$ satisfying $(\mathrm{H} 1)$ and $\|h\|_{\infty} \leq \gamma$.

Proof. Let $u=t+v$. We distinguish two cases: (i) $\|\nabla v\|_{2} \leq \eta|t|$ and (ii) $\|\nabla v\|_{2}>\eta|t|$. If (i) holds, then by (2.3) we get

$$
J(u) \geq \frac{|t|^{q+1}}{q+1} \alpha-C_{1} \int_{\Omega} u^{2} d x
$$

for some constant $C_{1}>0$. The existence of $C_{1}$ follows easily from assumption (F1). We now observe that if $\|u\|_{V}^{2}=\|\nabla v\|_{2}^{2}+t^{2}=s^{2}$, then $t^{2} \geq s^{2} /\left(1+\eta^{2}\right)$. Thus by (2.2) we have

$$
J(u) \geq \frac{\alpha s^{q+1}}{(q+1)\left(1+\eta^{2}\right)^{(q+1) / 2}}-C_{1} \max \left(1+K^{2},|\Omega|\right) s^{2} .
$$


Taking

$$
s_{\circ}=\left[\frac{\alpha}{2 C_{1}(q+1)\left(1+\eta^{2}\right)^{(q+1) / 2} \max \left(1+K^{2},|\Omega|\right)}\right]^{1 /(1-q)}
$$

we derive the estimate

$$
J(u) \geq \frac{\alpha s^{q+1}}{2(q+1)\left(1+\eta^{2}\right)^{(q+1) / 2}}
$$

for all $0<s \leq s_{\circ}$ and $\|u\|_{V}=s$. In case (ii) we have

$$
\begin{aligned}
\int_{\Omega} h|u|^{q+1} d x & \leq\|h\|_{\infty}|\Omega|^{(1-q) / 2}\|u\|_{2}^{q+1} \\
& \leq\|h\|_{\infty}|\Omega|^{(1-q) / 2} \max \left(1+K^{2},|\Omega|\right)^{(q+1) / 2}\|u\|_{V}^{q+1}
\end{aligned}
$$

and

$$
\|u\|_{V} \leq\|\nabla v\|_{2}\left(1+1 / \eta^{2}\right)^{1 / 2}
$$

Hence

$$
\begin{aligned}
J(u) \geq & \frac{\|u\|_{V}^{2}}{2\left(1+1 / \eta^{2}\right)}-\|h\|_{\infty}|\Omega|^{(1-q) / 2} \max \left(1+K^{2}, \Omega \mid\right)^{(q+1) / 2}\|u\|_{V}^{q+1} \\
& -\int_{\Omega} F(x, u) d x .
\end{aligned}
$$

Let $2<p<2^{*}=2 N /(N-2)$. By assumption (F1) there exists a constant $C_{2}>0$ such that

$$
F(x, t) \leq \frac{t^{2}}{4\left(1+1 / \eta^{2}\right) \max \left(1+K^{2},|\Omega|\right)}+C_{2} t^{p} \quad \text { for } t \geq 0
$$

Hence by the Sobolev embedding theorem we have

$$
\begin{aligned}
J(u) \geq & \frac{\|u\|_{V}^{2}}{4\left(1+1 / \eta^{2}\right)}-\|h\|_{\infty}|\Omega|^{(1-q) / 2} \max \left(1+K^{2},|\Omega|\right)^{(q+1) / 2}\|u\|_{V}^{q+1} \\
& -C_{3}\|u\|_{V}^{p}
\end{aligned}
$$

for some constant $C_{3}>0$. Let

$$
a=\frac{1}{4\left(1+1 / \eta^{2}\right)}, \quad b=|\Omega|^{(1-q) / 2} \max \left(1+K^{2},|\Omega|\right)^{(q+1) / 2}
$$

and

$$
k(t)=\|h\|_{\infty} b t^{q-1}+C_{3} t^{p-2} \quad \text { for } t \geq 0 .
$$

Then

$$
J(u) \geq\|u\|_{V}^{2}\left(a-k\left(\|u\|_{V}\right)\right)
$$

Since

$$
\inf _{t \geq 0} k(t)=\|h\|_{\infty^{\frac{p-2}{p-q}}} C_{5}=k\left(t_{\circ}\right)
$$


where

$t_{\circ}=\left[\frac{(1-q)\|h\|_{\infty} b}{C_{3}(p-2)}\right]^{\frac{1}{p-q-1}}, \quad C_{5}=C_{4}^{\frac{q-1}{p-q-1}}\left(b+C_{3} C_{4}\right)$ with $C_{4}=\frac{(1-q) b}{(p-2) C_{3}}$, we obtain, for $\|u\|_{V}=t_{\circ}$,

$$
J(u) \geq a t_{\circ}^{2} / 2
$$

provided $\|h\|_{\infty} \leq\left(\frac{a}{2 C_{5}}\right)^{\frac{p-q-1}{p-2}}$. Since $t_{\circ}$ depends on $\|h\|_{\infty}$ we may choose $\gamma \leq\left(\frac{a}{2 C_{5}}\right)^{\frac{p-q-1}{p-2}}$ so that $t_{\circ} \leq s_{\circ}$ for $\|h\|_{\infty} \leq \gamma$. If $\|u\|_{V}=\varrho=t_{\circ}$ and

$$
\kappa=\min \left(\frac{a t_{\circ}^{2}}{2}, \frac{\alpha s_{\circ}^{q+1}}{2(q+1)\left(1+\eta^{2}\right)^{(q+1) / 2}}\right)
$$

the estimate (2.4) easily follows.

We now observe that there exists an $e \in H^{1}(\Omega)$ such that $\|e\|_{V}>\varrho$ and $J(e)<0$. Indeed, it is enough to choose $\tau>0$ sufficiently large so that

$$
F(x, s) \geq l s^{2} / 2 \quad \text { for } s \geq \tau \text {. }
$$

Then we set $e(x)=t$ with $t \geq \tau$ sufficiently large so that

$$
J(t)=\frac{t^{q+1}}{q+1} \int_{\Omega} h(x) d x-\frac{l t^{2}}{2}|\Omega|<0
$$

We now define

$$
c=\inf _{g \in \Gamma} \max _{s \in[0,1]} J(g(s))
$$

where

$$
\Gamma=\left\{g \in C\left([0,1], H^{1}(\Omega)\right) ; g(0)=0, g(1)=e\right\} .
$$

TheOREM 2.2. Problem (1.1) has a mountain-pass solution provided (H1), (F1) and $\|h\|_{\infty} \leq \gamma$ hold.

Proof. The mountain-pass level generates a Palais-Smale sequence $\left\{u_{m}\right\} \subset H^{1}(\Omega)$, that is,

$$
J\left(u_{m}\right) \rightarrow c \quad \text { and } \quad J^{\prime}\left(u_{m}\right) \rightarrow 0 \quad \text { in } H^{-1}(\Omega) .
$$

First, we show that $\left\{u_{m}\right\}$ is bounded in $H^{1}(\Omega)$. In the contrary case we may assume that $\left\|u_{m}\right\| \rightarrow \infty$. Set $w_{m}=u_{m} /\left\|u_{m}\right\|$. It is clear that we can assume that $w_{m} \rightarrow w$ in $L^{q+1}(\Omega), w_{m} \rightarrow w$ in $H^{1}(\Omega)$ and $w_{m}(x) \rightarrow w(x)$ a.e. on $\Omega$. We claim that $w \not \equiv 0$ on $\Omega$. If $w \equiv 0$ on $\Omega$, then

$$
\left\|\nabla w_{m}\right\|_{2}^{2}=\frac{1}{\left\|u_{m}\right\|^{1-q}} \int_{\Omega} h(x)\left|w_{m}\right|^{q+1} d x+\int_{\Omega} p\left(x, u_{m}\right) w_{m}^{2} d x+o(1),
$$

where

$$
p(x, s)= \begin{cases}f(x, s) / s & \text { if } s \neq 0 \\ 0 & \text { if } s=0\end{cases}
$$


Since $p(x, s)$ is bounded, we see that $\left\|\nabla w_{m}\right\|_{2} \rightarrow 0$ and hence $\left\|w_{m}\right\| \rightarrow 0$. This leads to a contradiction as $\left\|w_{m}\right\|=1$ for each $m$. Therefore $w \not \equiv 0$ on $\Omega$. Let $\phi \in C^{1}(\bar{\Omega})$. It then follows from the $(\mathrm{PS})_{c}$ condition that

$$
\begin{aligned}
\int_{\Omega} \nabla w_{m} \nabla \phi d x-\frac{1}{\left\|u_{m}\right\|^{1-q}} \int_{\Omega} h(x)\left|w_{m}\right|^{q-1} w_{m} \phi d x & \\
& -\int_{\Omega} p\left(x, u_{m}\right) w_{m} \phi d x=o(1) .
\end{aligned}
$$

Since $w \not \equiv 0$, it follows from assumption $(\mathrm{F} 1)$ that $p\left(x, u_{m}\right) \rightarrow v$, where $v$ is given by $v=l_{1} \chi_{w>0}+l_{2} \chi_{w<0}$ and $w v$ is positive on a set of positive measure. Letting $m \rightarrow \infty$ in (2.7) we get

$$
\int_{\Omega} \nabla w \nabla \phi d x=\int_{\Omega} v w \phi d x
$$

for each $\phi \in C^{1}(\bar{\Omega})$ and consequently for each $\phi \in H^{1}(\Omega)$, so

$$
-\Delta w=v(x) w \geq 0 \quad \text { on } \Omega
$$

in a weak sense. Testing this equation with $\phi \equiv 1$ yields $\int_{\Omega} v w d x=0$, which is impossible. Thus $\left\{u_{m}\right\}$ is bounded in $H^{1}(\Omega)$. It is easy to show that $\left\{u_{m}\right\}$ is relatively compact in $H^{1}(\Omega)$ (that is, $u_{m} \rightarrow u$ in $H^{1}(\Omega)$ up to a subsequence) and $u$ is a solution of problem (1.1). By Theorem 10 in [3] we may assume that $u \geq 0$ on $\Omega$. The fact that $u>0$ on $\Omega$ follows from the maximum principle. Since $h$ changes sign on $\Omega$ and $f(x, s)>0$ for $s \neq 0$, the solution $u$ is not constant.

We point out here that Theorem 2.2 as well as Proposition 2.1 remain true if $h \leq 0$ on $\Omega$ and $h<0$ on a set of positive measure. The assumption that $h$ changes sign is needed to obtain a second solution (see Theorem 2.3 in Section 3). Then

If $h$ changes sign on $\Omega$ there exists $\varphi \in C^{1}(\bar{\Omega})$ with $\operatorname{supp} \varphi \subset\{x ; h(x)>0\}$.

$$
J(t \varphi)=\frac{t^{2}}{2} \int_{\Omega}|\nabla \varphi|^{2} d x-\frac{t^{q+1}}{q+1} \int_{\Omega} h(x)|\varphi|^{q+1} d x-\int_{\Omega} F(x, t \varphi) d x .
$$

According to assumption (F1), $F$ has a quadratic growth. Hence for sufficiently small $t>0$ we get $J(t \varphi)<0$. Thus $c^{*}=\inf _{\|u\| \leq \varrho} J(u)<0$ and $J(u)>0$ for $\|u\|=\varrho$. Using the Ekeland variational principle we are led to the second existence theorem:

TheOREm 2.3. Suppose that (H1) and (F1) hold and $\|h\|_{\infty} \leq \gamma$. Then problem (1.1) has a second solution satisfying $J(u)<0$.

We now consider the situation where $f$ is only asymptotically linear at $+\infty$. In this case we assume that $h<0$ on $\Omega$. We impose the following assumption on $f$ : 
$\left(\mathrm{F}^{+}\right) \quad f \in C(\bar{\Omega}, \mathbb{R}), f(x, 0)=0$ on $\Omega, f(x, s)>0$ on $\Omega$ for $s>0$ and

$$
\lim _{s \rightarrow \infty} \frac{f(x, s)}{s}=l>0 .
$$

We use the truncated functional

$$
J^{+}(u)=\frac{1}{2} \int_{\Omega}|\nabla u|^{2} d x-\frac{1}{q+1} \int_{\Omega} h(x)|u|^{q+1} d x-\int_{\Omega} F\left(x, u^{+}\right) d x,
$$

where $s^{+}=\max (0, s)$. This functional has a mountain-pass structure: there exist constants $\kappa>0, \varrho>0$ and $\gamma>0$ such that (2.4) holds.

Theorem 2.4. Let $h<0$ on $\Omega$. Suppose that $\left(\mathrm{F}^{+}\right)$holds and that $\|h\|_{\infty} \leq \gamma$. Then problem (1.1) has a solution.

Proof. We use a version of the mountain-pass principle that guarantees the existence of a Palais-Smale sequence $\left\{u_{m}\right\}$ (in the sense of Cerami): $J^{+}\left(u_{m}\right) \rightarrow c$ and $\left(1+\left\|u_{m}\right\|\right) J^{+\prime}\left(u_{m}\right) \rightarrow 0$ in $H^{-1}(\Omega)$ (see [4], [5] or [10]). It is enough to show that $\left\{u_{m}\right\}$ is bounded in $H^{1}(\Omega)$. Arguing by contradiction, assume that $\left\|u_{m}\right\| \rightarrow \infty$. Put $w_{m}=u_{m} /\left\|u_{m}\right\|$. We now repeat the argument from the proof of Theorem 2.2 and show that a weak limit $w$ in $H^{1}(\Omega)$ of $\left\{w_{m}\right\}$ is nonzero. If $w<0$ on a set of positive measure then $u_{m} \rightarrow-\infty$ on a set of positive measure. If we test $J^{+\prime}\left(u_{m}\right)$ with $u_{m}^{-}$, Fatou's lemma yields

$$
-\left\langle J^{+\prime}\left(u_{m}\right), u_{m}^{-}\right\rangle=\int_{\Omega}\left|\nabla u_{m}^{-}\right|^{2} d x+\int_{\Omega}(-h)\left|u_{m}^{-}\right|^{q+1} d x \rightarrow \infty,
$$

which is impossible. We now modify the definition of $p(x, s)$ by setting

$$
p(x, s)= \begin{cases}f(x, s) / s & \text { if } s>0, \\ 0 & \text { if } s \leq 0 .\end{cases}
$$

We may assume that $p\left(x, u_{m}\right) \rightarrow v$ in $L^{2}(\Omega)$. Obviously $w v>0$ on a set of positive measure. Testing the corresponding equation (2.8) with $\phi \equiv 1$, we arrive at a contradiction. Therefore $\left\{u_{m}\right\}$ is bounded in $H^{1}(\Omega)$ and the result follows.

3. Case $l_{1}=\infty$. We now turn our attention to the case $l_{1}=\infty$. In this situation we shall assume that $h<0$ on $\Omega$. Additionally, we assume that

$$
\begin{aligned}
& \lim _{s \rightarrow 0} \frac{f(x, s)}{s}=0, \quad \lim _{s \rightarrow \infty} \frac{f(x, s)}{s}=\infty, \\
& f(x, s) / s \text { is nondecreasing on }(0, \infty) \text { and } \\
& \lim _{s \rightarrow \infty} \frac{f(x, s)}{s^{p}}=0 \text { for some } 1<p<\frac{N+2}{N-2} .
\end{aligned}
$$

A solution in this case will be found as a critical point of the truncated functional $J^{+}$introduced in Section 2. It is clear that inequality (2.4) con- 
tinues to hold. Therefore we can establish the mountain-pass geometry for the functional $J^{+}$: there exist constants $\kappa_{1}, \varrho_{1}, \gamma_{1}>0$ such that $J^{+}(u) \geq \kappa_{1}$ for all $\|u\|=\varrho_{1}$ and $\|h\|_{\infty} \leq \gamma_{1}$.

Lemma 3.1. Suppose that $h<0$ on $\Omega$ and that (F2) holds. Let $\left\{u_{m}\right\} \subset$ $H^{1}(\Omega)$ be a sequence satisfying

$$
\left\langle J^{+}\left(u_{m}\right), u_{m}\right\rangle \rightarrow 0 \quad \text { as } m \rightarrow \infty .
$$

Then for every $t>0$, up to a subsequence,

$$
J^{+}\left(t u_{m}\right) \leq \frac{t^{2}+1}{2 m}+\left[\frac{t^{2}}{2}-\frac{t^{q+1}}{q+1}\right] \int_{\Omega} h(x)\left|u_{m}\right|^{q+1} d x+J^{+}\left(u_{m}\right) .
$$

The proof is similar to that of Proposition 4.1 in [8] and is omitted. We point out here that Proposition 4.1 in [8] has been proved for $\left\{u_{m}\right\} \in H_{0}^{1}(\Omega)$ (for the Dirichlet problem) but this result can be extended easily to the situation considered in this paper.

Theorem 3.2. Suppose that $h<0$ on $\Omega$ and that (F2) holds. Then for every $h$ satisfying $\|h\|_{\infty} \leq \gamma_{1}$, problem (1.1) has a mountain-pass solution.

Proof. We follow the ideas of [8]. It follows from the comments in the paragraph preceding Theorem 3.2 that the functional $J^{+}$has a mountainpass structure. Therefore there exists a sequence $\left\{u_{m}\right\} \subset H^{1}(\Omega)$ such that $J^{+}\left(u_{m}\right) \rightarrow c$, with $c>0$, and $\left(1+\left\|u_{m}\right\|\right) J^{+\prime}\left(u_{m}\right) \rightarrow 0$ in $H^{-1}(\Omega)$. It is sufficient to show that $\left\{u_{m}\right\}$ is bounded in $H^{1}(\Omega)$. In the contrary case we may assume that $\left\|u_{m}\right\| \rightarrow \infty$. Put $w_{m}=2 \sqrt{c} u_{m} /\left\|u_{m}\right\|$ and $t_{m}=2 \sqrt{c} /\left\|u_{m}\right\|$. We may assume that $w_{m} \rightarrow w$ in $H^{1}(\Omega), w_{m} \rightarrow w$ in $L^{r}(\Omega), 1 \leq r<2^{*}$, and $w_{m} \rightarrow w$ a.e. on $\Omega$. First we check that $w \neq \equiv 0$. Indeed, if $w \equiv 0$, then

$$
\int_{\Omega}\left|w_{m}\right|^{q+1} d x \rightarrow 0 \text { and } \int_{\Omega} F\left(x, w_{m}^{+}\right) d x \rightarrow 0
$$

and $\left\|w_{m}\right\|^{2}=\left\|\nabla w_{m}\right\|_{2}^{2}+\left\|w_{m}\right\|_{2}^{2}=\left\|\nabla w_{m}\right\|_{2}^{2}+o(1)$, so

$$
J^{+}\left(w_{m}\right)=\frac{1}{2}\left\|w_{m}\right\|^{2}+o(1)=2 c+o(1) .
$$

We now apply Lemma 3.1 to show that

$$
\begin{aligned}
J^{+}\left(w_{m}\right) & =J\left(t_{m} u_{m}\right) \leq \frac{t_{m}^{2}+1}{2 m}+\left[\frac{t_{m}^{2}}{2}-\frac{t_{m}^{q+1}}{1+q}\right] \int_{\Omega} h(x)\left|u_{m}\right|^{q+1} d x+J^{+}\left(u_{m}\right) \\
& =\frac{t_{m}^{2}+1}{2 m}+\left[\frac{t_{m}^{1-q}}{2}-\frac{1}{q+1}\right] \int_{\Omega} h(x)\left|w_{m}\right|^{q+1} d x+J^{+}\left(u_{m}\right) \rightarrow c,
\end{aligned}
$$


which contradicts (3.1). Let $\Omega^{+}=\{x \in \Omega ; w(x)>0\}$. It is clear that $u_{m} \rightarrow \infty$ on $\Omega^{+}$. Repeating the argument from Theorem 2.2 we have

$$
\begin{aligned}
\int_{\Omega} \nabla w_{m} \nabla w d x-\frac{1}{\left\|u_{m}\right\|^{1-q}} \int_{\Omega} h(x)\left|w_{m}\right|^{q-1} w_{n} w d x & \\
& -\int_{\Omega} p\left(x, u_{m}^{+}\right) w_{m}^{+} w d x=o(1),
\end{aligned}
$$

where $p(x, t)$ is the function defined in the proof of Theorem 2.4. We then have

$$
\begin{aligned}
\int_{\Omega}|\nabla w|^{2} d x & =\lim _{m \rightarrow \infty} \int_{\Omega} \frac{f\left(x, u_{m}^{+}\right)}{u_{m}^{+}} w_{m}^{+} w^{+} d x \geq \lim _{m \rightarrow \infty} \int_{\Omega^{+}} \frac{f\left(x, u_{m}^{+}\right)}{u_{m}^{+}} w_{m}^{+} w d x \\
& \geq \int_{\Omega^{+}} \lim _{m \rightarrow \infty}\left[\frac{f\left(x, u_{m}^{+}\right)}{u_{m}^{+}} w_{m}^{+}\right] w d x .
\end{aligned}
$$

Since $\lim _{m \rightarrow \infty} \frac{f\left(x, u_{m}+\right)}{u_{m}^{+}} w_{m}^{+}=\infty$ on $\Omega^{+}$we deduce from this that $\left|\Omega^{+}\right|=0$ and $w(x) \leq 0$ a.e. on $\Omega$ with strict inequality on a set of positive measure. Hence $u_{m} \rightarrow-\infty$ on a set of positive measure. Testing $J^{+\prime}\left(u_{m}\right)$ with $u_{m}^{-}$ yields

$$
-\left\langle J^{+\prime}\left(u_{m}\right), u_{m}^{-}\right\rangle=\int_{\Omega}\left|\nabla u_{m}^{-}\right|^{2}+\int_{\Omega}(-h)\left|u_{m}^{-}\right|^{q+1} d x \rightarrow \infty,
$$

which is impossible.

4. The linear case $f(x, s)=\lambda s$. We conclude this paper with some observations on the linear case $f(x, s)=\lambda s$.

(I) If $h \geq 0$ on $\Omega$ with strict inequality on a set of positive measure, then problem (1.1) has no solution for $\lambda \geq 0$. However it admits at least one solution for $\lambda<0$.

If $u \geq 0$ is a solution of (1.1) with $\lambda \geq 0$, then

$$
\int_{\Omega} h(x) u^{q+1} d x+\lambda \int_{\Omega} u d x=0
$$

which yields $u \equiv 0$. If $\lambda<0$, then a solution can be obtained as a minimizer of the constrained minimization problem

$$
\inf \left\{\int_{\Omega}\left(|\nabla u|^{2}-\lambda u^{2}\right) d x ; u \in H^{1}(\Omega), \int_{\Omega} h(x)|u|^{q+1} d x=1\right\} .
$$

(II) If $h(x) \leq 0$ on $\Omega$ with strict inequality on a set of positive measure, then problem (1.1) admits at least one solution provided $\lambda>0$ is sufficiently small. This solution can be obtained by the mountainpass principle. 
Indeed, inspection of the proof of Proposition 2.1 shows that the functional

$$
I(u)=\frac{1}{2} \int_{\Omega}|\nabla u|^{2} d x-\frac{1}{q+1} \int_{\Omega} h(x)|u|^{q+1} d x-\frac{\lambda}{2} \int_{\Omega}\left(u^{+}\right)^{2} d x
$$

has a mountain-pass structure. This follows from estimate (2.3) and from the fact that $I(t)<0$ for $t>0$ large. To show the boundedness of the resulting Palais-Smale sequence we repeat the proof of Theorem 2.2. Obviously, in this case problem (1.1) has no solution for $\lambda \leq 0$.

We point out here that in case (II) equation (1.1) with the mixed boundary value conditions (or the Dirichlet boundary conditions) admits a solution for $\lambda$ greater than the first eigenvalue of the corresponding eigenvalue problem. For simplicity, let $\partial \Omega=\Gamma_{1} \cup \Gamma_{2}$ where $\Gamma_{1}$ is a closed and nonempty subset of $\partial \Omega$. We consider the problem

$$
\left\{\begin{array}{l}
-\Delta u=h u^{q}+\lambda u \quad \text { in } \Omega \\
\partial \nu / \partial \nu=0 \quad \text { on } \Gamma_{2}, \quad u=0 \quad \text { on } \Gamma_{1}, \quad u>0 \quad \text { on } \Omega .
\end{array}\right.
$$

We denote by $V_{\circ}^{1}(\Omega)$ the subspace of functions $u$ of $H^{1}(\Omega)$ such that $u=0$ on $\Gamma_{1}$ equipped with the norm $\|\nabla u\|_{2}$. Let $\lambda_{1}>0$ be the first eigenvalue of the problem

$$
\left\{\begin{array}{l}
-\Delta u=\lambda u \quad \text { in } \Omega \\
u=0 \quad \text { on } \Gamma_{1}, \quad \partial u / \partial \nu=0 \quad \text { on } \Gamma_{2} .
\end{array}\right.
$$

Proposition 4.1. If $h$ is a negative constant, then problem (1.1) admits at least one solution for every $\lambda>\lambda_{1}$.

Proof. First we observe that the function $k(t)=h t^{q}+\lambda t, 0 \leq t<\infty$, satisfies $k(t)<0$ for $0 \leq t<\delta_{\circ}=(-h / \lambda)^{1 /(1-q)}$ and $k(t)>0$ for $t>\delta_{\circ}$. Using this we check that the functional

$$
I(u)=\frac{1}{2} \int_{\Omega}|\nabla u|^{2} d x-\frac{h}{q+1} \int_{\Omega}\left(u^{+}\right)^{q+1} d x-\frac{\lambda}{2} \int_{\Omega}\left(u^{+}\right)^{2} d x
$$

has a mountain-pass structure. Indeed, we have

$$
\begin{aligned}
I(u)= & \frac{1}{2} \int_{\mathbb{R}^{N}}|\nabla u|^{2} d x-\int_{0 \leq u \leq \delta_{\circ}}\left(\frac{1}{q+1} h u^{q+1}+\frac{\lambda}{2} u^{2}\right) d x \\
& -\int_{u \geq \delta_{\circ}}\left(\frac{1}{q+1} h u^{q+1}+\frac{\lambda}{2} u^{2}\right) d x \\
\geq & \frac{1}{2} \int_{\Omega}|\nabla u|^{2} d x-\int_{u \geq \delta_{\circ}}\left(\frac{1}{q+1} h u^{q+1}+\frac{\lambda}{2} u^{2}\right) d x .
\end{aligned}
$$


With the aid of the Sobolev inequality we estimate the last integral:

$$
\begin{aligned}
\int_{u \geq \delta_{\circ}} u^{2}\left(\frac{\lambda}{2}+\frac{u^{q-1} h}{q+1}\right) d x & \leq\left(\frac{\lambda}{2}+\frac{\delta_{\circ}^{q-1} h}{q+1}\right) \int_{u \geq \delta_{\circ}} u^{2} d x \\
& =\frac{1}{\delta_{\circ}^{2^{*}-2}}\left(\frac{\lambda}{2}+\frac{\delta_{\circ}^{q-1} h}{q+1}\right) \int_{\Omega}|u|^{2^{*}} \\
& \leq C(\Omega)\left(\int_{\Omega}|\nabla u|^{2} d x\right)^{2^{*} / 2} .
\end{aligned}
$$

Combining the last two estimates we get

$$
I(u) \geq \frac{1}{2}\|\nabla u\|_{2}^{2}-C(\Omega)\|\nabla u\|_{2}^{2^{*}}
$$

Hence there exist constants $\varrho>0$ and $\kappa>0$ such that

$$
I(u) \geq \kappa \quad \text { for }\|\nabla u\|_{2}=\varrho .
$$

Let $\phi_{1}>0$ be the principal eigenfunction of the problem (4.2). Then $I\left(t \phi_{1}\right)<0$ and $\left\|\nabla\left(t \phi_{1}\right)\right\|_{2} \geq \varrho$ for $t>0$ sufficiently large. The result now follows from the mountain-pass principle.

\section{References}

[1] A. Ambrosetti and M. Struwe, Existence of steady vortex rings in an ideal fluid, Arch. Rat. Mech. Anal. 108 (1989), 97-109.

[2] D. Arcoya and S. Villegas, Nontrivial solutions for a Neumann problem with a nonlinear term asymptotically linear at $-\infty$ and superlinear at $+\infty$, Math. Z. 219 (1995), 499-513.

[3] H. Berestycki, I. Capuzzo-Dolcetta and L. Nirenberg, Variational methods for indefinite superlinear homogeneous elliptic problems, NoDEA 2 (1995), 553-572.

[4] G. Cerami, Un criterio di esistenza per i punti critici su varietà illimitate, Rend. Acad. Sci. Let. Ist. Lombardo 112 (1978), 332-336.

[5] - Sull'esistenza di autovalori per un problema al contorno non lineare, Ann. Mat. (4) 24 (1980), 161-179.

[6] G. B. Li and H. S. Zhou, Multiple solutions to $p$-Laplacian problems with asymptotic nonlinearity as $u^{p-1}$ at infinity, J. London Math. Soc. (2) 65 (2002), 123-138.

[7] - - - Asymptotically "linear" Dirichlet problem for p-Laplacian, Nonlinear Anal. 43 (2001), 1043-1055.

[8] S. J. Li, S. P. Wu and H. Zhu, Solutions to semilinear elliptic problems with combined nonlinearities, J. Differential Equations 185 (2002), 200-224.

[9] M. Lucia, P. Magrone and H.-S. Zhu, A Dirichlet problem with asymptotically linear and changing sign nonlinearity, Rev. Mat. Complut. 16 (2003), 465-481.

[10] M. Schechter, A variation of the Mountain Pass lemma and applications, J. London Math. Soc. 44 (1991), 491-502.

[11] C. L. Tang, Multiple solutions of Neumann problem for elliptic equations, Nonlinear Anal. 54 (2003), 637-650. 
[12] C. L. Tang, Solvability of Neumann problem for elliptic equations at resonance, ibid. 44 (2001), 323-335.

[13] H. S. Zhou, Existence of asymptotically linear Dirichlet problem, ibid. 44 (2001), 909-918.

[14] -, An application of a mountain pass theorem, Acta Math. Sinica English Ser. 18 (2002), 27-36.

Department of Mathematics

University of Queensland

St. Lucia 4072, Qld, Australia

E-mail: jhc@maths.uq.edu.au
Wuhan Institute of Physics and Mathematics

Chinese Academy of Sciences

P.O. Box 71010

Wuhan 430071, P.R. China

E-mail: jfyang@wipm.ac.cn 